Ann. Génét. Sél. anim., I974, 6 (2), 2II-2I7.

\title{
ÉTUDE D'UN GENE DE NANISME LIÉ AU SEXE CHEZ LA POULE : IMPORTANCE DE L'ÉTAT D'ENGRAISSEMENT ET GAIN DE POIDS CHEZ L'ADULTE
}

\author{
P. MÉRAT et F. H. RICARD* \\ avec la collaboration technique de G. MARChE* et de G. Coquerelle \\ Laboratoire de Génétique factorielle, \\ Centre national de Recherches zootechniques, I. N.R. A., \\ 78350 Jouy en Josas \\ * Station expérimentale d'Aviculture du Magneraud, I. N.R. A., \\ 17700 Surgères
}

\section{RÉSUMÉ}

Dans deux populations différentes, l'état d'engraissement de poules âgées de un an ou plus est plus faible chez les naines $\left(d w^{-}\right)$que chez les normales $\left(D w^{-}\right)$, contrairement à ce qui avait été observé chez les poulettes avant maturité sexuelle. En cours de ponte, le gain de poids des poules naines est inférieur à celui de leurs sœurs normales mais en valeur relative les différences ne sont pas significatives. L'explication du moindre engraissement des poules naines pourrait être une meilleure utilisation des nutriments énergétiques d'origine alimentaire en vue de la production des œufs chez les animaux porteurs du gène $d w$.

\section{INTRODUC'TION}

Un gène lié au sexe réduisant la taille, dw, décrit par HưTT (I959), a été retrouvé à plusieurs reprises, et divers chercheurs ont analysé ses effets. Parmi ceux-ci, une plus grande importance relative de la graisse abdominale chez le Poulet, suggérant une teneur accrue en lipides de la carcasse entière, a été notée (MÉRA'T et GuILLAumE, I969; OUHAYOUN, I970 ; RICARD, I970 ; RAJARA'TNAM et al., I97I).

Ces observations ont été faites à divers âges, mais toujours avant maturité sexuelle. A notre connaissance, la teneur en lipides corporels, ou le pourcentage du 
tissu gras abdominal qui lui est fortement lié (DELPECH et RICARD, I965; RICARD, données non publiées) n'ont pas été évalués chez des animaux adultes nains et normaux, en particulier chez la Poule en ponte. Tel est l'objet du présent article.

\section{MATÉRIEL E'T MÉTHODES}

Des données concernant le tissu gras abdominal et le gain de poids chez l'adulte ont été recueillies dans 2 échantillons différents de poules. Le premier est issu de la population expérimentale du Laboratoire de Génétique factorielle de Jouy en Josas. Divers gènes y sont maintenus en ségrégation à chaque génération, en particulier un gène de nanisme lié au sexe, issu d'une mutation initiale (MÉRAT, 1969) et que nous désignerons par $d w$ comme le gène décrit par HuTT. Le deuxième échantillon provient d'une population expérimentale de la Station du Magneraud, de format plus lourd et où le gène $d w$ est également maintenu en ségrégation. Dans les deux cas, l'aliment était donné ad libitum.

Dans un lot de poules de la population de Jouy en Josas, nées en septembre I97o, mises en cages individuelles de février à juillet $197 \mathrm{I}$, puis utilisées comme reproductrices au sol en aoutseptembre I97I, 22 couples de sœurs ou demi-sœurs, l'une de génotype $D w^{-}$, l'autre $d w^{-}$, ont été choisis. Ces poules ont été abattues à un an d’âge, le 15 septembre 1971, et leur tissu gras abdominal disséqué et pesé (dépôts localisés autour du gésier, de la glande de Fabricius et contre la paroi abdominale).

Chaque groupe de 22 poules normales ou naines était subdivisé en 11 poules hétérozygotes pour le gène "Cou nu " $(N a n a)$ et I I poules de plumage normal (na na). En l'absence d'effet significatif associé au locus $N a$ sur le pourcentage de graisse abdominale, nous avons regroupé les animaux quel que soit leur génotype à ce locus. L'aliment donné correspondait à une formule à 16 p. roo de protéines et environ 2520 Calories d'énergie métabolisable par $\mathrm{kg}$.

Dans cette même population, nous avons étudié la variation de poids de poules et de coqs adultes, placés en cages, en 1972 et 1973. En effet, on peut penser qu'une fraction importante du gain de poids des animaux adultes dans ces conditions est constituée par une augmentation des tissus gras : nous avons obtenu sur 30 poules de la même population, maintenues en cages entre les âges approximatifs de 6 et to mois et abattues à ce dernier âge, une corrélation de $+0,6 \mathbf{r}$ $(\mathbf{P}<0,01)$ entre leur gain de poids en cages et leur pourcentage de graisse abdominale à l'abattage (Mérat et Bordas, I972). En 1972, 56 poules $D w^{-}$et $57 d w^{-}$, sœurs ou demi-sœurs des premières, étaient placées en cages entre environ 6 et ro mois d'âge, et leur gain de poids durant cette période était enregistré. Il en est de même de 29 poules $D w^{-}$et $30 d w^{-}$en I973, de 23 coqs $D w d w$ et $29 d w d w$ des mêmes âges en 1972, en fin de 23 coqs $D w d w$ et $29 d w d w$ en r 973 .

Dans la population du Magneraud, 52 paires de poules sours, l'une normale et l'autre naine, ont été abattues le I 3 février 1973 à l'âge moyen de 6o semaines (extrêmes : 58-62 semaines). Ces poules ont toujours été élevées au sol et ont reçu un aliment contenant $16,5 \mathrm{p}$. I oo de protéines et 2 70o Calories d'énergie métabolisable par $\mathrm{kg}$, soit une ration un peu plus dense que celle des poules abattues à Jouy en Josas. Le tissu gras abdominal a été également disséqué et pesé. De plus, des pesées individuelles ont été faites à 24 et 36 semaines ainsi que la veille de l'abattage.

Dans chacun des deux échantillons, le rapport du poids de la graisse abdominale au poids corporel mesuré immédiatement avant abattage et après un jeûne d'environ I 6 heures a été comparé pour les poules des deux génotypes par un test de Student, selon la méthode des couples. La variable utilisée a une distribution de fréquences s'écartant quelque peu de la normalité (dissymétrie avec étalement plus grand vers les valeurs élevées) et sa variance est significativement plus grande pour les poules de génotype normal $D w^{-}$que pour les naines ; cependant, ces écarts aux conditions idéales d'utilisation du test $\mathbf{t}$ ont paru suffisamment limités pour que l'emploi de ce test soit possible.

Dans la population de Jouy en Josas, les gains de poids ont été comparés d'abord en valeur absolue pour les deux génotypes dans chaque sexe. Cette comparaison a été faite en constituant des couples de sours ou demi-sours (ou frères ou demi-frères), l'une naine et l'autre normale. Un test de Student-Fisher était fait sur les différences entre les deux membres de chaque couple pour l'ensemble des deux années, un écart à la normalité et une différence de variance entre génotypes pour le gain de poids ayant paru suffisamment modérés pour que le test soit applicable. Sur les mêmes couples d'animaux, nous avons également comparé, par la même méthode, les gains de poids exprimés en p. Ioo du poids corporel de chaque animal en début d'expérience. Ici encore, les conditions ont paru suffisantes pour permettre l'application d'un simple test $t$ (en particulier, la distribution de fréquences apparaît un peu plus proche de la normalité que pour les gains 
exprimés en valeur absolue). Dans l'échantillon du Magneraud, les gains de poids ont été calculée d'une part entre 24 et 36 semaines et d'autre part entre 36 semaines et la veille de l'abattags (6o semaines en moyenne). Comme pour l'échantillon de Jouy en Josas, les comparaisons entre poules normales et naines ont porté sur les gains exprimés en valeurs brutes et sur les gains exprimés en pourcentage du poids en début de période ( 24 ou 36 semaines).

En dehors de l'état d'engraissement et du gain de poids, nous connaissions les performances zootechniques de ces animaux, en particulier poids moyen de l'œuf (à l'âge de 36 semaines pour l'échantillon de Jouy et celui du Magneraud) et le nombre d'œufs (de l'entrée en ponte à l'âge de 57 semaines pour l'échantillon du Magneraud et à l'âge de 40 semaines pour celui de Jouy).

\section{RÉSULTATS}

Les résultats concernant la graisse abdominale sont présentés dans le tableau $\mathbf{I}$. Dans les deux populations, les poules naines apparaissent sensiblement moins grasses. La différence en pourcentage entre les poules normales et les naines y est du même ordre. Elle approche du seuil 5 p. Ioo de signification pour la population de Jouy ( $t=\mathrm{I}, 95$ avec $2 \mathrm{I}$ d. 1.) et est significative au seuil I $\mathrm{p}$. Iooo pour les animaux du Magneraud ( $t=3,75$ pour $5 \mathrm{I}$ d. 1.).

\section{TABLEAU I}

Poids et pourcentage comparés du tissu gras abdominal chez des poules adultes normales $\left(D w^{-}\right)$et naines $\left(d w^{-}\right)$

\begin{tabular}{|c|c|c|c|c|}
\hline \multirow{2}{*}{ Caractéristique } & \multicolumn{2}{|c|}{ Poules $D w^{-}$} & \multicolumn{2}{|c|}{ Poules $d w^{-}$} \\
\hline & $\bar{x}$ & $s$ & $\bar{x}$ & $s$ \\
\hline 1. Population de Jouy en Josas & \multicolumn{4}{|c|}{$(\mathrm{N}=22$ couples $)$} \\
\hline Poids vif avant abattage $(\mathrm{g})$. & 2385 & 330 & 1551 & 231 \\
\hline Poids tissu gras abdominal $(\mathrm{g})$ & 88 & 56 & 42 & 27 \\
\hline Tissu gras/Poids vif $(\%) \ldots \ldots \ldots$ & 3,58 & 1,90 & 2,60 & 1,20 \\
\hline 2. Population du Magneraud & \multicolumn{4}{|c|}{$(\mathrm{N}=52$ couples $)$} \\
\hline Poids vif avant abattage $(\mathrm{g})$ & 2999 & 335 & 2006 & 222 \\
\hline Poids tissu gras abdominal $(\mathrm{g})$ & 170 & 73 & 88 & 36 \\
\hline Tissu gras/Poids vif $(\%) \ldots \ldots$ & 5,56 & 2,12 & 4,34 & 1,53 \\
\hline
\end{tabular}

Les résultats concernant les gains de poids en valeur absolue et en pourcentage du poids vif en début de période sont présentés dans le tableau 2. Seul le total des deux années (I972 et 1973) est donné en ce qui concerne la population de Jouy en Josas, les résultats étant de même sens chaque année.

Dans la population de Jouy en Josas, le gain de poids est inférieur pour les poules naines, tant en valeur absolue que par rapport au poids corporel de départ. Pour les coqs, par contre, il y a peu de différence en valeur absolue, mais en pourcentage, les nains gagnent plus de poids. Le test de comparaison des gains de poids absolus, sur 74 couples de poules et 38 couples de coqs formés sur l'ensemble des deux années, donne une valeur $t=4,09(\mathrm{P}<0,00 \mathrm{I})$ pour les premières et $t=0,2 \mathrm{I}(\mathrm{NS})$ 
pour les seconds. Quant aux gains en p. Ioo du poids corporel, sur les mêmes couples, on obtient $t=\mathrm{I}, 59(\mathrm{P} \simeq 0, \mathrm{Io})$ pour les poules et $t=-2,39(\mathrm{P}<0,05)$ pour les coqs. Dans le premier cas, d'ailleurs, le test d'un écart à l'hypothèse nulle dans le sens particulier (et inattendu) d'une supériorité des poules normales donnerait une probabilité proche de 0,05 .

TABLEAU 2

Gains de poids comparés pour des poules et des coqs adultes normaux et nains

\begin{tabular}{|c|c|c|c|c|}
\hline \multirow[b]{2}{*}{ Animaux } & \multicolumn{2}{|c|}{ Normaux } & \multicolumn{2}{|c|}{ Nains } \\
\hline & $\begin{array}{l}\text { gain brut } \\
\text { (g) }\end{array}$ & $\begin{array}{l}\text { gain } \\
\text { relatif } \\
(\%)\end{array}$ & $\begin{array}{l}\text { gain brut } \\
\text { (g) }\end{array}$ & $\begin{array}{l}\text { gain } \\
\text { relatif } \\
(\%)\end{array}$ \\
\hline \multicolumn{5}{|l|}{ 1. Population de Jouy en Josas } \\
\hline Poules $(\mathbf{1}) \quad \ldots \ldots \ldots \ldots \ldots \ldots$ & 71,7 & 2,91 & 36,1 & 2,22 \\
\hline Coqs $\left({ }^{2}\right) \ldots \ldots \ldots \ldots$ & 42,5 & 1,66 & 44,0 & 2,57 \\
\hline \multicolumn{5}{|l|}{$\begin{array}{c}\text { 2. Population du Magneraud } \\
\text { (poules) }\end{array}$} \\
\hline Gain $24-36$ semaines $\left({ }^{3}\right) \ldots \ldots$ & 170,0 & 6,33 & 157,3 & 8,44 \\
\hline Gain $36-60$ semaines $\left({ }^{3}\right) \ldots \ldots$ & 191,5 & 6,50 & 107,3 & 5,37 \\
\hline
\end{tabular}

(1) Moyennes calculées sur 85 poules normales $\left(D w^{-}\right)$et 87 poules naines $\left(d w^{-}\right)$. $(d w d w)$

(2) Moyennes calculées sur 46 coqs normaux (Dwdw) et 58 coqs nains

$\left(^{3}\right)$ Moyennes calculées sur 52 poules normales $\left(D w^{-}\right)$et 52 poules naines $\left(d w^{-}\right)$.

Dans la population du Magneraud, le gain de poids moyen est inférieur, en valeur brute, chez les poules naines, comme pour l'échantillon de Jouy en Josas. Mais la différence n'est significative que pour la période $36-60$ semaines $(t=0,37$ pour la période $24-36$ semaines, $t=2,05$ pour la période 36-6o semaines). En valeur relative, le gain de poids des poules normales est un peu plus faible que celui des poules naines entre 24 et 36 semaines. Il est un peu plus fort entre 36 et 60 semaines, mais les différences ne sont pas significatives $(t=\mathrm{I}, 53$ pour la première période $; t=\mathrm{I}, \mathrm{I} 4$ pour la seconde).

\section{DISCUSSION ET CONCLUSIONS}

Le fait que la graisse abdominale, tant en valeur absolue que relativement, soit plus réduite chez les poules naines que chez leurs sœurs normales, est en opposition avec ce qui a été observé chez le jeune. Ce résultat semble susceptible d'être généralisé puisque nous l'avons observé dans deux populations différentes, ne recevant pas le même aliment, l'une plus légère et placée en cages, 1'autre plus lourde et élevée au sol. Or, le pourcentage des dépots gras abdominaux est fortement lié à la teneur en lipides corporels totaux. Sur un échantillon de 30 pondeuses de type Rhode $\times$ Wyandotte 
âgées de 57 semaines, nous avons observé une corrélation de 0,92 entre ces deux variables (RICARD, données non publiées).

Une première illustration de cet engraissement plus faible des poules naines pourrait être donnée en comparant les gains de poids au cours de la période de ponte. Les résultats du tableau 2 suggèrent un gain de poids moindre chez les poules naines pour la population de Jouy en Josas et la période 36-60 semaines dans le cas de la population du Magneraud. Mais les différences ne sont pas significatives quand on s'intéresse aux valeurs relatives : les différences de gain de poids ne peuvent donc expliquer qu'en partie les différences d'engraissement. En ce qui concerne les coqs de la population de Jouy en Josas, les différences de gain de poids vont en sens contraire, mais nous ne disposons pas, pour ces animaux, de données sur l'importance du tissu gras abdominal.

Une deuxième explication d'un engraissement moindre des poules naines peut être recherchée dans une différence des processus physiologiques de la ponte. On saî́ que les nutriments énergétiques d'origine alimentaire fournissent la majeure partie des lipides du vitellus (LECLERCQ, I973). Par ailleurs, GUILLAUME (I973) a observé une lipolyse plus faible chez les poules naines que chez les poules normales. On peut alors supposer que les poules naines mobilisent moins vite leurs réserves lipidiques pour la ponte, qu'elles les reconstituent encore moins vite, mais qu'elles utilisent une part plus importante des nutriments énergétiques de la ration pour leur synthèse du vitellus. En ce qui concerne la ponte proprement dite, quoique la vitellogenèse des poules naines paraisse moins intense que celle des poules de taille normale (JAAP et Mohammadian, I969), le poids de vitellus qu'elles forment en un temps donné est plus important, relativement à leur poids corporel, que pour les poules normales. L'intensité de ponte des naines pouvant être estimée à $80-90 \mathrm{p}$. Ioo et le poids de leur jaune à 90-95 p. roo des valeurs correspondantes pour les poules de génotype $D w^{-}$, le produit de ces deux pourcentages est de l'ordre de 72 à 85 p. Ioo, alors que le poids

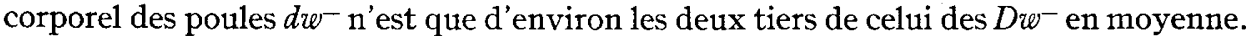
Ce raisonnement peut être illustré, à titre d'exemple, par les résultats rassemblés dans le tableau 3 et concernant l'échantillon du Magneraud. Le poids vif des poules

TABLEAU 3

Production d'cufs comparée pour des poules normales et naines (échantillon du Magneraud)

\begin{tabular}{|c|c|c|c|}
\hline Caractéristiques & Poules $D w^{-}$ & Poules $d w$ & $\begin{array}{c}\text { Rapport } \\
\text { nain/normal } \\
(\%)\end{array}$ \\
\hline Poids vif 24 semaines $(g) \ldots$ & 2875 & 1904 & 66 \\
\hline Nbre d'œufs pondus à 57 sem. & 160,5 & 140,9 & 88 \\
\hline $\begin{array}{l}\text { Poids moy. de l'œuf à } 36 \text { sem. } \\
\qquad \text { (g) } \ldots \ldots \ldots \ldots \ldots \ldots \ldots\end{array}$ & 56,9 & 53,0 & 93 \\
\hline $\begin{array}{l}\text { Masse d'œufs exportée à } 57 \\
\text { sem. }(\mathrm{g}) \ldots \ldots \ldots \ldots \ldots \ldots \ldots\end{array}$ & 9138 & 7462 & 82 \\
\hline Rapport $\frac{\text { Masse exportee }}{\text { Poids vif } 24 \text { sem. }}$. & 3,2 & 3,9 & 123 \\
\hline
\end{tabular}


naines en début de ponte représente $66 \mathrm{p}$. roo du poids de leurs sœurs normales. Mais pour la masse d'œufs exportée (nombre d'œufs à 57 semaines $\times$ poids moyen de l'œuf à 36 semaines), le rapport est de 82 p. Ioo. Autrement dit, les poules normales de l'échantillon étudié ont exporté une masse d'œufs égale à 3,2 fois leur poids en début de ponte, tandis que pour les poules naines ce rapport s'élève à 3,9. Dans la population de Jouy, nous observons une différence qui va dans le même sens.

Nous avançons donc 1'hypothèse que les poules naines en fin de ponte sont moins grasses que les poules normales parce qu'elles ont utilisé les nutriments énergétiques d'origine alimentaire pour leur synthèse du jaune plutôt que pour les stocker sous forme de réserves lipidiques. Sur le plan zootechnique, une aptitude inférieure des pondeuses reproductrices naines à l'engraissement peut contribuer, au moins en partie, à leur meilleur rendement alimentaire et rendre leur carcasse plus appréciée à la réforme. Par ailleurs, si leur plus faible teneur corporelle en lipides inclut les dépôts gras superficiels, ceci pourrait peut-être aussi jouer un rôle secondaire dans leur meilleure tolérance à la chaleur suggérée par ailleurs (MÉRAT et BORDAS, sous presse).

Reģu pour publication en mai 1974.

\title{
REMERCIEMENTS
}

Nous remercions M. J. Guillaume, Station de Recherches avicoles, 37 - Nouzilly, de ses utiles suggestions et critiques à la lecture de ce manuscrit.

\section{SUMMARY}

\author{
STUDY OF A SEX-LINKED DWARF GENE IN THE FOWL : \\ FATNESS AND WEIGH'T INCREASE IN ADULTS
}

In two different populations, dwarf $\left(d w^{-}\right)$hens aged I year or more are less fat than normals $\left(D w^{-}\right)$, conversely to the observed situation among pullets before sexual maturity. During egg laying, the increase in weight of dwarf hens is lower than that of their normal sisters, but in relative value the differences are not significant. An explanation of the lower fatness of dwarf hens could be a better utilization of feed energy for egg production among birds carrying the $d w$ gene.

\section{RÉFÉRENCES BIBLIOGRAPHIQUES}

Delpech P., Ricard F. H., I965. Relation entre les dépôts adipeux viscéraux et les lipides corporels chez le Poulet. Ann. Zootech., 14, I8I-189.

Guillaume J., I973. Effets du gène de nanisme récessif lié au sexe, $d w$, sur le métabolisme énergétique de la poule. VI ${ }^{\mathbf{e}}$ symposium sur le métabolisme énergétique, Stuttgart, Publication E. A.A.P. $\mathrm{n}^{\circ}$ I4 (sous presse).

Hutt F. B., I959. Sex-linked dwarfism in the fowl. J. Hered., 50, 209-22x.

JaAP R. G., Mонамmadian M., 1969. Sex-linked dwarfism and egg production of broiler dams. Poult. Sci., 48, 344-346.

Leclerco B., 1973. Contribution de l'aliment et des réserves corporelles à la genèse des lipides vitellins de la poule. Thèse doctorat d'État, Fac. Sci., Paris. 
Mérat P., I969. Étude d'un gène de nanisme lié au sexe chez la Poule. I. Description sommaire et performances. Ann. Génét. Sél. anim., 1, 19-26.

Mérat P., Bordas A., I972. Consommation alimentaire de pondeuses blanches (Ii) et colorées (ii). Ann. Génét. Sél. anim., 4, 375-384.

Mérat P., Bordas A., I974. Effets associés aux gènes $d w$ (nanisme) et $N a$ (cou nu) chez la Poule sur la production d'œufs et la consommation alimentaire à deux températures. Ann Génét. Sél. anim. (sous presse).

Mérat P., Guillaume J., I969. Étude d'un gène de nanisme lié au sexe chez la Poule. II. Fonctionnement thyroïdien. Ann. Génét. Sél. anim., 1, I3I-I33.

Ouhayoun J., i97o. Étude d'un gène de nanisme lié áu $\mathrm{s} \approx \mathrm{xe}$ chez la Poule. V. Composition corporelle in vivo. Ann. Génét. Sél. anim., 2, 33-36.

Rajaratnam G., Summers J. D., Wood A. S., Muran E. T. Jr., I97i. Some physiological and nutritional aspects of the dwarf chicken. Can. J. Anim. Sci., 51, 209-2 6 .

Ricard F. H., r97o. Étude d'un gène de nanisme lié au sexe chez la Poule. IV. Observation sur la croissance et les caractéristiques de carcasse du jeune poulet. Ann. Génét. Sél. anim., 2, r9-31. 